\title{
Excitonic Energy Shifts in CdMnTe/CdMgTe Quantum Wells under Resonant Excitation in Presence of 2D Carrier Gas
}

\author{
A. Trajnerowicz ${ }^{a}, \mathrm{~A} \cdot \mathrm{GolniK}^{a}, \mathrm{P} \cdot \mathrm{KossaCki}^{a}$, \\ W. BardyszeWski ${ }^{b}$, M. Wiater ${ }^{c}$, G. KARCZEWSKi $^{c}$ \\ AND T. WoJTOWICZ ${ }^{c}$ \\ ${ }^{a}$ Institute of Experimental Physics, University of Warsaw \\ Hoża 69, 00-681 Warsaw, Poland \\ ${ }^{b}$ Institute of Theoretical Physics, University of Warsaw \\ Hoża 69, 00-681 Warsaw, Poland \\ ${ }^{c}$ Institute of Physics, Polish Academy of Sciences \\ al. Lotników 32/46, 02-668 Warsaw, Poland
}

\begin{abstract}
Systematic studies of amplitude and energy changes of excitonic lines in a strong excitation regime were carried out by a pump-probe method. The series of samples containing quantum wells with well width from $80 \AA$ to $140 \AA$ was investigated. One $80 \AA$ sample was $n$-doped with iodine, the rest of the quantum wells were intentionally undoped but contained a $2 \mathrm{D}$ gas of free holes. Its density could be varied by changing an intensity of additional illumination. The resonant creation of high population of e1hh1 excitons causes the energetic blue shift of the line due to interactions between excitons. The blue shift did not depend on the concentration of $2 \mathrm{D}$ gas of carriers whereas it did depend on the power of excitation beam. Model calculations of absorption show qualitative agreement with the experimental data.
\end{abstract}

PACS numbers: 78.67.De, 71.35.Lk, 78.47.-p, 78.47.J-

\section{Introduction}

The fast developing and expanding technology creates a requirement for new materials and devices based on lower dimension structures, especially for 
semiconductors in the telecommunications and computing industries. To create good materials, and quantum structures based on such structures, the behavior of quasiparticles defining properties of such materials must be deeply understood. Of key importance from the technological point of view in semiconductors are such quasiparticles as electrons and holes and in lower dimensions their complexes, such as excitons and trions (charged excitons). In spite of years of studies on CdTe 2D materials there are many mechanisms, especially many-body effects, to be understood, such as interactions between ground and higher excitonic states in presence or absence of free carrier gas. Until now, some work has been done regarding interactions among trions, neutral e1hh1 excitons and 2D gas of free carriers [1-6], with even higher excitonic states studied in a specially designed multi-quantum well (MQW) structure [7], but there is a wide field of phenomena to be clearly understood. One of such phenomenon is an origin of energy shift of the exciton transition after creation a high population of the excitons and an influence of the free carriers on this energy shift. In the present work we demonstrate and discuss the influence of population of free carriers on the behavior of the heavy holes (e1hh1) excitons. Furthermore, we conducted model theoretical calculations and compared them with our experimental data.

\section{Samples and experiment}

The reflectivity signal was measured in the pump-probe experiment. The beam from $\mathrm{Ti}^{3+}: \mathrm{Al}_{2} \mathrm{O}_{3}$ tunable 60 fs pulse laser was split into more intense pump, and weaker - probe beams. The pump was spectrally filtered in a dispersive set containing a diffracting grating. Time between the pump and the probe pulses was electronically controlled by a precise mechanical delay line. To control the free carrier gas density an additional illumination (a tungsten halogen lamp) was used. The reflected signal was focused on a front slit of $300 \mathrm{~mm}$ monochromator and recorded by a CCD camera. The picosecond pump pulse of spectral full width at half maximum (FWHM) of about $1 \mathrm{~nm}$ and controlled mean power up to $1 \mathrm{~mW}$ on the spot of about $250 \mu \mathrm{m}$ diameter created resonantly a density of heavy-holes excitons up to a few $\times 10^{10}$ per $\mathrm{cm}^{2}$.

Several $(\mathrm{Cd}, \mathrm{Mn}) \mathrm{Te} /(\mathrm{Cd}, \mathrm{Mg}) \mathrm{Te}$ quantum well samples with different well width and $500 \AA$ barriers were studied. The width of QWs varied from $80 \AA$ to $140 \AA$. The samples were intentionally undoped showing $p$-type conductivity (positively charged trions). One sample with the QW $80 \AA$ wide was $n$-doped with iodine [8]. All samples were grown by MBE on GaAs substrate, therefore only measurements of reflectivity were available without mechanical interference into samples' structure. Manganese doping of about $0.6-0.7 \%$ for $p$-type samples and $0.21 \%$ for $n$-doped sample allowed to identify excitonic transitions and the sign of charge carriers in 2D gas through the giant Zeeman splitting. Because of a barrier width close to $500 \AA$ all studied samples revealed absorptive-like reflectance spectra [9]. The samples were mounted stress-free in a cryostat with a superconducting 
magnet and immersed in pumped liquid helium. The He bath temperature was between $1.5 \mathrm{~K}$ and $1.7 \mathrm{~K}$. In case of zero magnetic field measurements only a mutual orientation of circular polarizations of the pump and the reflected probe pulses is important, therefore we will only state whether the pulses were co-polarized or cross-polarized.

\section{Results}

The measurements were performed for different free-carrier densities, different densities of created excitons and different samples.

After resonant excitation of the exciton state by the intense pump pulse we observe the energy shift of the line towards higher energies, so-called blue shift. It results from the complex interactions between $2 \mathrm{D}$ carrier gas, spin polarized neutral excitons (carriers) obtained by pump pulse and excitons related to probe pulse. This interaction involves screening, phase space filling and energy renormalization due to many-body effects.

In Fig. 1 the energy shifts of the exciton transition for $100 \AA$-type QW are plotted versus delay time for four different free carrier densities at fixed power of the pump for co- and cross-polarization configurations. The shifts were obtained by fitting the Gaussian absorption profiles to the reflectivity spectra. The free-carrier density was controlled by changing of the intensity of the additional illumination by changing of halogen lamp voltage. The values of the densities were obtained from linear dependence of the carrier density from the ratio of the charged exciton intensity to the intensity of neutral one [10].

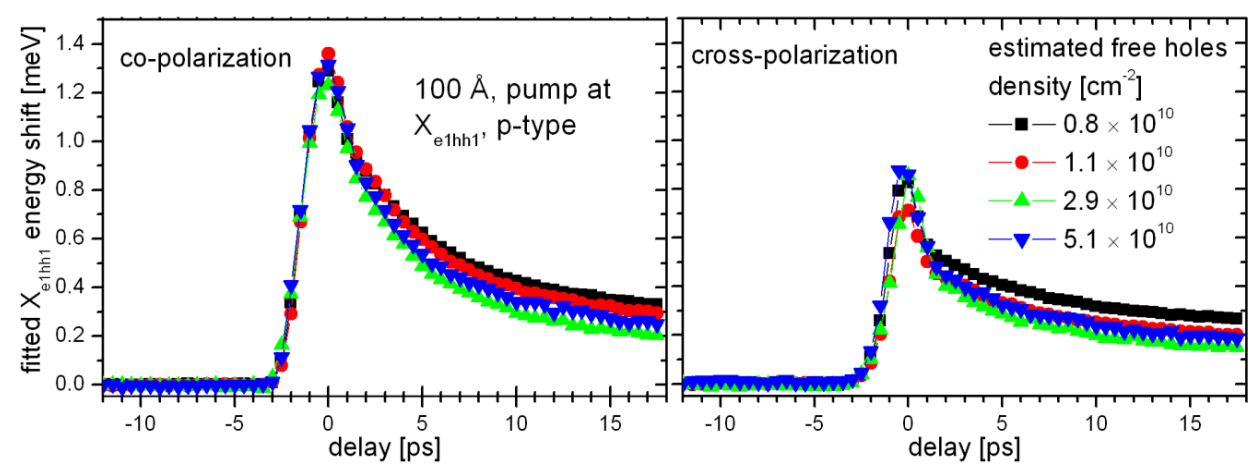

Fig. 1. $\quad X_{\mathrm{e} 1 \mathrm{hh} 1}$ time dependent energy shifts obtained from fitting the reflectivity spectra for $100 \AA \mathrm{QW}$ for four different free-carrier densities for fixed pump power for coand cross-polarization configurations.

In the moment of creation of the large population of excitons the significant energy shifts are observed for all free-carrier densities. Although the energy of the exciton transition changes from one $2 \mathrm{D}$ gas density to another, the blue shifts stay the same. 
For this experiment an increase in the amplitude of the exciton transitions was observed (not shown), which originates from differences in absorption of the material for different free carrier densities [4]. Also there are time dependent changes in the amplitude which differ for different 2D free carrier densities especially in cross-configuration.

In Fig. 2 the dependence of energy shifts on delay time is displayed for the same sample but in conditions of the same 2D gas density for three different powers of excitation beam (different populations of resonantly created heavy-hole excitons) for both polarization configurations. In this case strong exciton density dependence is observed. The energy shift decreases with decreasing population of created excitons.

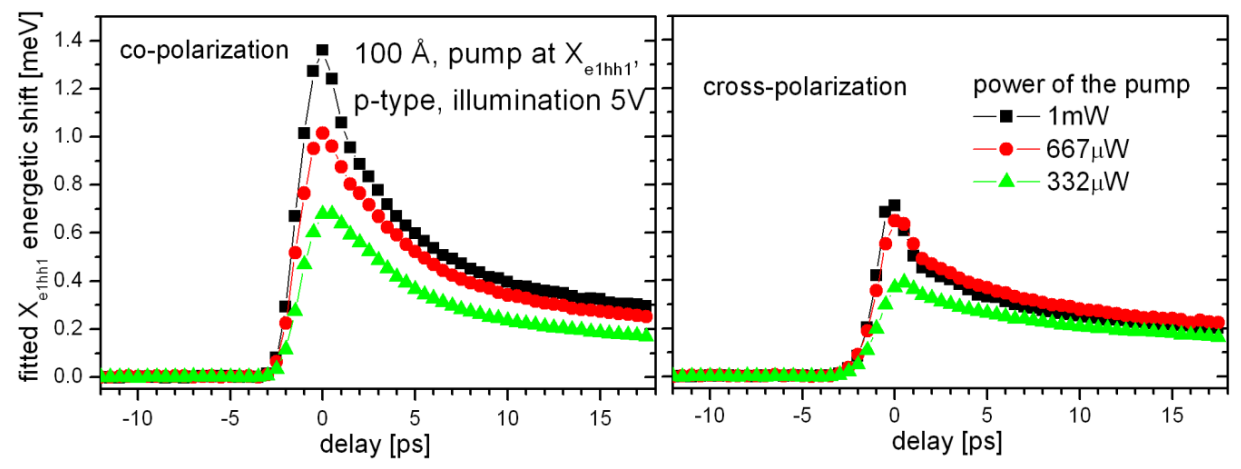

Fig. 2. $X_{\mathrm{e} 1 \mathrm{hh} 1}$ time dependent energy shifts obtained from fitting the reflectivity spectra for $100 \AA \mathrm{QW}$ for three different pump powers at fixed free-carrier density for coand cross-polarization configurations.

Also in this experiment differences in the amplitudes are seen (not shown), but in this case the larger power of excitation beam is, the amplitude of the transition is smaller (exciton transition oscillator strength weakens). Probably it is caused by the rise of the sample temperature. The time dependent changes in the amplitude seem not to depend on the pump power.

\section{Theoretical model calculations}

In order to calculate the absorption spectra we have solved the equation of motion for the electron-hole pair correlation function, taking into account the static screening of the Coulomb potential and the Pauli blocking term [11]. We have incorporated $k p$ and Coulomb mixing between the mini-bands in quantum wells [12]. The Coulomb coupling between free carriers is accounted for by renormalization of the one-particle energy bands within the shielded Hartree-Fock approximation in the static limit. Non-equilibrium distributions of carriers with different spins are assumed to model spin polarized systems in time resolved photoluminescence experiments. We assume that both spin subsystems are character- 
ized by the same temperature in the valence and conduction band. The BetheSalpeter equation is solved by discretizing the radial relative momentum of the electron and the hole and diagonalizing the non-hermitian effective Hamiltonian matrix [13]. Typical calculation taking into account 5 topmost valence mini-bands and using 384 mesh points of the discrete momenta requires diagonalization of a sparse $3840 \times 3840$ matrix. We have employed Lanczos technique in which the problem is reduced to a diagonalization of a $300 \times 300$ tridiagonal matrix. The computation time with respect to the conventional diagonalization is thus reduced by a factor of 25 .

The level of spin polarization for electrons and holes in the time resolved circularly polarized photoluminescence experiment depends on the delay time and on the spin relaxation times. In our preliminary study we have simply fitted the level of spin polarization of both types of carriers and the concentration of excess pairs to the experimental spectra. A more thorough model which would take into account the spin kinetics in the systems is needed in order to fully interpret these experiments.

\section{Discussion}

The behavior of energy shifts presented in Fig. 1 for $100 \AA$ QW are either well reproduced for other samples which makes us to believe that that kind behavior is also independent of the QW width. The independence of the blue shifts of free-carrier density indicates that the interactions between created excitons are not influenced by the existence of the free carriers. Here, it is worth emphasizing that the samples were intentionally undoped and the free carriers in the QW are the surface doped ones [14], and their density is rather small (of about a few $\times 10^{10} \mathrm{~cm}^{-2}$ ). The free-carrier density decreases fast with increase in additional illumination and in some cases it was hard to estimate the density value due to a lack of visible and analyzable charged exciton transition. In spite of the small density of the free carriers, the dependence of the neutral exciton amplitude was well observable. It increases with decreasing $2 \mathrm{D}$ gas of carrier concentration which results from changes of the absorption. Moreover, the time dependent changes of amplitude also differ for different free-carrier populations. It could originate from changes in the oscillator strength of exciton transition. For cross-configuration this effect is stronger which is caused by additional phenomenon, oscillator strength stilling (OSS) by created biexcitonic states.

Preliminary theoretical calculations (see Fig. 3) of absorption either show the independence of energy shifts of the free-carrier density for both $p$-type and $n$-type doping. It has to be emphasized that those calculations overestimate the band-gap renormalization effect which shows up as a shift of absorption lines towards lower energies where in the experiment we observe the blue shifts.

In the second case (Fig. 2) where we fixed 2D gas density (about $10^{10} \mathrm{~cm}^{-2}$ ) and changed the power of the pump different behavior of the blue shifts is clearly 


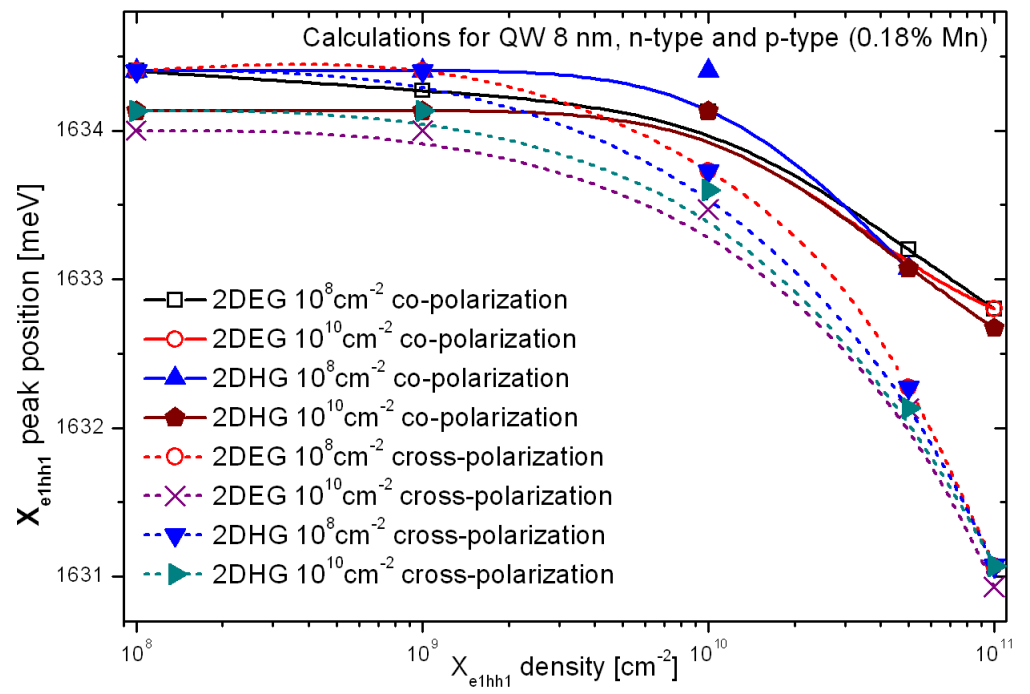

Fig. 3. Calculated $X_{\mathrm{e} 1 \mathrm{hh} 1}$ peak positions for $n$-type and $p$-type $80 \AA \mathrm{QW}$ as a function of $X_{\mathrm{e} 1 \mathrm{hh} 1}$ density for two different free-carrier densities and for co- and cross-polarization configurations.

visible. The larger population of created excitons is, the larger is the energy shift, which proves the origin of the shift, which are interactions between excitons. This is also seen in calculated data where with increasing exciton density the increase in the shift is noticed.

In that experiment we see the drop off of the exciton amplitude with a rising of excitation power, which may be caused by a growing temperature of the sample. There are no effects on the time dependent changes of the amplitude.

\section{Conclusions}

Several (Cd, Mn)Te/(Cd, Mg)Te quantum wells with different width and types of free carriers were studied by means of the femtosecond pump-probe method. The reflectivity spectra were taken for resonantly excited e1hh1 transition in conditions of different free-carrier densities by the same excitation power and for different excitation power for fixed free-carrier density. These lines were analyzed by fitting the Gaussian profiles and then time dependences of the energy shift of the exciton and its amplitude were discussed. The blue shifts of this transition were observed, both for co- and cross-polarization configurations of the pump and probe beams. The shifts are free carrier independent and depend only on the population of created excitons, which originates from the interaction between excitons. 2D gas of free carriers influences the oscillator strength of the transition. Taking into account overestimation of the band-gap renormalization effect of the calculations, there is good qualitative agreement between experimental data and theoretical calculations. 


\section{Acknowledgments}

This work was partially supported by the Polish Ministry of Science and Higher Education as research grants in years 2006-2010, grant NN202328134 (GR 2617), and also by the E.U. grant MTKD-CT-2005-029671.

\section{References}

[1] P. Kossacki, J. Cibert, D. Ferrand, Y. Merle d'Aubigné, A. Arnoult, A. Wasiela, S. Tatarenko, J.A. Gaj, Phys. Rev. B 60, 16018 (1999).

[2] P. Płochocka, P. Kossacki, W. Maślana, J. Cibert, S. Tatarenko, C. Radzewicz, J.A. Gaj, Phys. Rev. Lett. 92, 177402 (2004).

[3] P. Kossacki, H. Boukari, M. Bertolini, D. Ferrand, J. Cibert, S. Tatarenko, J.A. Gaj, B. Deveaud, V. Ciulin, M. Potemski, Phys. Rev. B 70, 195337 (2004).

[4] P. Kossacki, J. Phys. C 15, R471 (2003), and references therein.

[5] M.T. Portella-Oberli, V. Ciulin, J.H. Berney, B. Deveaud, M. Kutrowski, T. Wojtowicz, Phys. Rev. B 69, 235311 (2004).

[6] P. Kossacki, P. Płochocka, B. Piechal, W. Maślana, A. Golnik, J. Cibert, S. Tatarenko, J.A. Gaj, Phys. Rev. B 72, 035340 (2005).

[7] H. Rahimpour Soleimani, S. Cronenberger, M. Gallart, P. Gilliot, J. Cibert, O. Cregut, B. Honerlage, J.-P. Likforman, Appl. Phys. Lett. 87, 192104 (2005).

[8] M. Kutrowski, T. Wojtowicz, P. Kossacki, V. Ciulin, J. Kossut, Phys. Status Solidi B 229, 791 (2002).

[9] X.L. Zheng, D. Heiman, B. Lax, F.A. Chambers, Appl. Phys. Lett. 52, 287 (1998).

[10] W. Maślana, P. Kossacki, J.A. Gaj, D. Ferrand, M. Bertolini, S. Tatarenko, J. Cibert, M. Kutrowski, T. Wojtowicz, Acta Phys. Pol. A 110, 255 (2006).

[11] M.J. Snelling, P. Perozzo, D.C. Hutchings, L. Galbraith, A. Miller, Phys. Rev. B 49, 17160 (1994-II).

[12] W. Bardyszewski, D. Yevick, Yong Liu, C. Rolland, S. Bradshaw, J. Appl. Phys. 80, 1136 (1996).

[13] W. Bardyszewski, D. Yevick, M. Prywata, Phys. Rev. B 62, 1835 (2000-I).

[14] W. Maślana, P. Kossacki, M. Bertolini, H. Boukari, D. Ferrand, S. Tatarenko, J. Cibert, J.A. Gaj, Appl. Phys. Lett. 82, 1875 (2003). 